appearance of several cephalosporins with similar activity this area of therapy is fast becoming a prescriber's nightmare.

The concluding chapter, written by Dr Finland, gives the reader insight into the changing behaviour of Pseudomonas in hospital practice over a 50-year timespan.

In summary, this is a readable book with a wealth of references accompanying each section, and at a reasonable price it should be accessible to all hospital workers who confront $P$. aeruginosa. The only critical comments to be made are the absence of an index, making cross-reference difficult, and the occasional unnecessary duplication in related chapters.

\section{Renal Pathology}

By E. M. Darmady and Angus G. Maciver. Pp. 548, illustrated. Postgraduate Pathology Series. Butterworths, London, Boston, Sydney, Wellington, Durban, Toronto, 1980. $£ 35.00$.

This most recent volume of Sir Theo Crawford's Postgraduate Pathology Series reconfirms the status of renal pathology as an established subspeciality. Recruits to the ranks of hospital pathologists must be prepared to meet the challenge of subspeciality demands, and conversely, practising nephrologists have a responsibility to seek an informed opinion on their histological problems.

This rapprochement of pathology and clinical medicine in the subspecialities is reflected in the organization of this volume, in which as much space is devoted to description of the clinical features of various renal diseases as to their strictly histopathological aspects. To some extent this is a disadvantage, for even though the book has 548 pages, several major problems are dealt with rather summarily. There is, for example, no discussion of the technical aspects of fixation, cutting or staining of biopsy sections for light or electron microscopy and no reference to thin sectioning of epoxy resin-embedded samples, though microdissection and immunofluorescence techniques are discussed in detail. The glomerulonephritides, which appear to dominate clinicopathological meetings, are accorded only $20 \%$ of the overall space, and the difficult problem of the coarsely scarred kidney and its association with vesico-ureteric reflux receives fewer pages than the amino-acidaemias.
The chapters on renal anatomy and development are very helpful, and Professor Darmady's personal interest in micro-ब dissection leads to an interesting new viewpoint on many subjects.

The presentation is in general good, and referencing is helpfully copious. The black and white photographic repro? ductions however, are not always adequate to demonstrate important features, particularly of the glomerular lesions

\section{Sixth Report on the World Health Situation 1973-77}

Part I Global Analysis, Pp. vii + 290; Part II Review byes Country and Area, Pp. vi +412 . World Health Organiza- $\overrightarrow{0}$ tion, Geneva, 1980. Part I, Sw. fr. 20.00. Part II, Sw. fr:28.00.

About twice in a decade the World Health Organization issues a report on the world situation. The sixth such repord covering the period 1973-77 became available in 1980. This is a grand strategy document and examines problems world wide. In accord with most of WHO's work more emphasis is placed on health in developing countries. The first volumeis is concerned with general issues and will be of interest to? students of international health. It will also be a guide tow ministers and one they can use in discussion with governmentP colleagues. The period under review was one in which freshi emphasis was placed on primary health care, and this is brought out together with the overall importance on health of social and behavioural factors and particularly poverty. Greater emphasis is now placed on family health and rather less on the great tropical diseases of malaria, schistosomiasis and the like. The second volume is a review by country and area. What is missed out is almost as interesting as whabiso included. The writers were clearly under great constraint: for example, the report on China with 1000 million people $(a$ quarter of the world's population) is contained in $2 \frac{1}{2}$ pases and sandwiched between Australia with a population of ${ }^{+} a$ mere 13 million which has 4 pages and the Cook Islands which received $1 \frac{1}{2}$ pages for their population of 30000 .

These volumes will be useful reference books for those⿳亠丷厂 working in the international health field and their contents are a tribute to the many international civil servants of the World Health Organization who collect, correct and collate infor $\frac{3}{3}$ mation. 